\title{
INTEGRAL REPRESENTATION OF CONTINUOUS FUNCTIONS $\left({ }^{1}\right)$
}

\author{
BY \\ WALTER RUDIN

\section{INTRODUCTION}

It was shown by F. Riesz $[5 ; 350]\left({ }^{2}\right)$ that every subharmonic function $u$ can be represented as the sum of the potential of its mass distribution plus a harmonic function; the potential appears in the form of a Stieltjes integral (Riesz's theorem is stated in (2.2.1)). We prove that the Stieltjes integral may be replaced by a Lebesgue integral if $u$ is continuous, and if the lower generalized Laplacian of $u$ is less than $+\infty$, except possibly on a set of capacity zero (Theorem II). In other words, the above assumptions imply the absolute continuity of the mass distribution associated with $u$.

We obtain this result as a consequence of Theorem I, which deals with the representation of continuous functions in integral form. In another paper, Theorem I will be used in an investigation of the uniqueness theory for Laplace series. The theorem was actually suggested by this application, and is of a type similar to a theorem of Zygmund $[9 ; 276]$ on the representation of continuous functions of one variable.

Our results are stated for the plane, but analogous theorems evidently hold for continuous functions of three or more variables, if the generalized Laplacians are suitably defined (see, for instance, [7]).

1.1. Notation. Let $D$ be a finite plane domain (that is, a connected open set not containing the point at infinity). Let $Z$ be a closed and bounded plane set of capacity zero (see 2.3). Let $J(P, r)$ denote the closed circular disc bounded by the circle $C(P, r)$ with center at $P$, and radius $r$. If the function $F \in L$ on $C(P, r)$, we put

$$
\Delta_{r} F(P)=\frac{1}{2 \pi r} \int_{C(P, r)} F(Q) d s_{Q}-F(P) .
$$

If there exists $r_{0}>0$ such that $F \in L$ on $C(P, r)$ for $0<r<r_{0}$, we define the generalized Laplacian of $F$ at $P$ by

Presented to the Society, September 2, 1949; received by the editors May 21, 1949 and, in revised form, November 2, 1949.

(1) The present paper is based on Part I of the author's thesis, Uniqueness theory for Laplace series, submitted in partial fulfillment of the requirements for the degree of Doctor of Philosophy in the Graduate School of Arts and Sciences of Duke University. The author wishes to acknowledge his indebtedness to Professor J. J. Gergen, under whose direction the thesis was written.

$\left({ }^{2}\right)$. Numbers in brackets refer to the bibliography at the end of the paper. 


$$
\Lambda F(P)=\lim _{r \rightarrow 0} 4 \Delta_{r} F(P) / r^{2},
$$

provided the limit exists. The upper and lower Laplacians, $\Lambda^{*} F(P)$ and $\Lambda_{*} F(P)$, are defined likewise, with lim sup and lim inf in place of lim.

By Wiener's sequence method $[8 ; 25]$ it is possible to define Green's function for any bounded domain $R$. We therefore do not have to restrict ourselves to Dirichlet domains. We shall introduce some more notation in 3.1.

1.2. Theorem I. Let the function $F$ be continuous in $D$. Suppose

(i) $\Lambda^{*} F(P)>-\infty, \Lambda_{*} F(P)<+\infty$ for $P$ in $D-D \cdot Z$;

(ii) there exists a function $y$, defined in $D$, such that $y \in L$ on every closed subset of $D$, and such that $y(P) \leqq \Lambda^{*} F(P)$ for $P$ in $D$.

Then

(a) $\Lambda F(P)$ is finite at almost all points $P$ of $D$;

(b) $\Lambda F \in L$ on every closed subset of $D$;

(c) at almost all points $P$ of $D$ we have

$$
\iint_{J(P, r)}|\Lambda F(Q) \log P Q| d Q<+\infty \quad(J(P, r) \subset D)
$$

(d) at all points $P$ of $D$ at which (1.2.1) holds, and for all bounded domains $R$ which contain $P$ and whose closure is contained in $D$, we have

$$
F(P)=-\frac{1}{2 \pi} \iint_{R} \Lambda F(Q) g(P, Q) d Q+H(P),
$$

where $g(P, Q)$ is Green's function for $R$, and $H$ is harmonic in $R$ and assumes the values of $F$ on the boundary of $R$ (in the sense of Wiener [8;25], if $R$ is not a Dirichlet domain).

1.3. Theorem II. Let the function $u$ be continuous and subharmonic in $D$. Suppose that $\Lambda_{*} u(P)<+\infty$ for $P$ in $D-D \cdot Z$. Then, at all points $P$ of $D$, and for all bounded domains $R$ which contain $P$ and whose closure is contained in $D$, we have

$$
u(P)=-\frac{1}{2 \pi} \iint_{R} \Lambda u(Q) g(P, Q) d Q+H(P),
$$

where $g(P, Q)$ is Green's function for $R$, and $H$ is the least harmonic majorant of $u$ in $R$. In addition, if $D$ is bounded, and if $u$ has a harmonic majorant in $D$, then (1.3.1) holds if $R=D$.

Evidently, (1.2.2) (and (1.3.1)) may also be written in the form

$$
F(P)=\frac{1}{2 \pi} \iint_{R} \Lambda F(Q) \log P Q d Q+h(P),
$$


in which the integral represents the potential of a mass distribution of density $\Lambda F$, and $h$ is harmonic in $R$.

\section{Subharmonic Functions, SETS OF CAPACity Zero}

2.1. Definition. A function $u$ is said to be subharmonic in $D$ if $[4 ; 333]$

(a) $u$ is upper semi-continuous in $D$,

(b) $u(P)<+\infty$ in $D$,

(c) $u(P)$ is finite on a dense set in $D$,

(d) for every domain $G$ with boundary $B$, such that $G+B \subset D$, the inequality $u(P) \leqq H(P)$ on $B$ implies $u(P) \leqq H(P)$ in $G$ for every function $H$ which is harmonic in $G$ and continuous on $G+B$.

The condition (d) may be replaced by

$$
\Delta_{r} u(P) \geqq 0
$$

provided $J(P, r) \subset D[4 ; 334]$, or by

$$
\Lambda^{*} u(P) \geqq 0
$$

for $P$ in $D[3 ; 14]$.

2.2. Lemma. If $u$ is subharmonic in $D$, then $\Lambda u(P)$ is finite at almost all points $P$ of $D$, and $\in L$ on every closed subset of $D$.

Proof. There exists a non-negative set function $\mu$, called the mass distribution of $u$, such that (1) $\mu(e)$ is finite for any Borel set $e$ contained with its closure in $D,(2)$ if $G$ is any open set contained with its boundary in $D$, then $\mu$ is absolutely additive on $G$, and

$$
u(P)=\iint_{G} \log P Q d \mu_{Q}+h(P)
$$

where $h$ is harmonic in $G[5 ; 350],[7 ; 452]$.

Defining the symmetric derivative $[6 ; 149]$ by

$$
D_{\mathrm{sym}} \mu(P)=\lim _{r \rightarrow 0} \frac{1}{\pi r^{2}} \mu(J(P, r)),
$$

we have, by the two-dimensional case of a theorem of Saks [7],

$$
\Lambda u(P)=2 \pi D_{\mathrm{sym}} \mu(P)
$$

at all points $P$ at which $D_{\mathrm{sym}} \mu(P)$ is finite.

Since $\mu$ is additive, the derivative $D \mu(P)$ (and, a fortiori, $D_{\text {sym }} \mu(P)$ ) is finite p. p. and $\in L$ on $E$, where $E$ is any measurable set for which $\mu(E)$ is finite.

Combining these results, we obtain the lemma.

2.3. Sets of capacity zero. Let $E$ be a closed and bounded plane set whose complement contains an unbounded domain $U$, and which is contained in the interior $I$ of a circle $C$. Let $\Gamma$ be the boundary of $U$. Put $V=U \cdot I$. Then 
$V$ is a domain. Let $u$ be the function which is harmonic in $V$ and which is the sequence solution $[8 ; 25]$ of the Dirichlet problem, corresponding to the boundary values 1 on $\Gamma$ and 0 on $C$.

The set $E$ is said to be of capacity zero if the function $u$ thus determined vanishes in $V[1 ; 208]$. This definition is independent of the choice of $C$.

Closed and bounded plane sets of capacity zero, which we shall denote by the letter $Z$, have the following geometric properties [1]:

(2.3.1) If $C$ is a circle, then $Z \cdot C$ is of measure zero on $C$.

(2.3.2) If $G$ is a domain, then $G-G \cdot Z$ is a domain.

We shall also make use of the following result:

(2.3.3) Let $G$ be a domain; Let $Z \subset G$; let $u$ be subharmonic in $G-Z$ and bounded above in a neighborhood of $Z$. If we define $u(P)$ on $Z$ by

$$
u(P)=\lim _{Q \rightarrow P, Q \in Z} u(Q),
$$

then the extended function is subharmonic in $G[2 ; 31]$.

The following lemma will enable us to infer that the function $H$ in (1.2.2) and (1.3.1) is harmonic.

2.4. Lemma. Let $E$ be a set of measure zero, contained in the finite domain $R$. Let $F$ be defined on $R-E$. Suppose $F \in L$ on $J(P, r)$ if $J(P, r) \subset R$, and suppose $\Delta_{r} F(P)=0$ for $P$ on $R-E$, if $J(P, r) \subset R$. Then there exists a function $H$ which is harmonic in $R$, such that $H(P)=F(P)$ on $R-E$.

Proof. It is easily verified that the function

$$
H(P)=H_{r}(P)=\frac{1}{\pi r^{2}} \iint_{J(P, r)} F(Q) d Q \quad(P \in R ; J(P, r) \subset R)
$$

is independent of $r$ for fixed $P$ on $R-E$, coincides with $F(P)$ on $R-E$, is continuous in $R$, hence independent of $r$ for fixed $P$ on $E$. It follows that $H$ has the Gaussian mean value property for all $J(P, r) \subset R$.

\section{THE OPERATOR $\Omega$}

It will be convenient to have a short symbol for integrals of the type appearing in (1.2.2) and (1.3.1). Hence we make the following definition.

3.1. Definition. Let $f \in L$ on $R$, where $R$ is a bounded domain. Put $f^{+}=\max (f, 0), f^{-}=-\min (f, 0)$. We define

$$
\Omega f(P)=\Omega_{R} f(P)=-\frac{1}{2 \pi} \iint_{R} f(Q) g(P, Q) d Q
$$

at all points $P$ of $R$ at which at least one of the integrals

$$
\iint_{R} f^{+}(Q) g(P, Q) d Q, \quad \iint_{R} f^{-}(Q) g(P, Q) d Q
$$


is finite. $g(P, Q)$, as before, is Green's function for $R$. It will not be necessary in the sequel to indicate the dependence of $\Omega$ on $R$.

Let $J(P, r) \subset R$, and suppose $f \in L$ on $R$. We shall use the following properties of $\Omega$ :

3.2. $\Omega f^{+}$and $\Omega f^{-}$are subharmonic in $R$.

3.3. $\Omega f \in L$ on $C(P, r)$, and $\Omega f \in L$ on $J(P, r)$; hence $\Omega f(P)$ is finite at almost all points $P$ of $R$.

3.4. If $f$ is bounded above in $R$, then $\Omega f$ is lower semi-continuous and $>-\infty$ in $R$.

3.5. If $P$ is a point at which $\Omega f(P)$ is defined, then

$$
\begin{gathered}
\Delta_{r} \Omega f(P)=\frac{1}{2 \pi} \iint_{J(P, r)} f(Q) \log \frac{r}{P Q} d Q, \\
\lim _{r \rightarrow 0} \frac{1}{2 \pi r} \int_{C(P, r)} \Omega f(Q) d s_{Q}=\Omega f(P) .
\end{gathered}
$$

3.6. At almost all points $P$ of $R, \Lambda \Omega f(P)=f(P)$.

3.7. If $f$ is upper semi-continuous and less than $+\infty$ at $P$, then $\Lambda^{*} \Omega f(P)$ $\leqq f(P)$.

3.8. If $f$ is bounded above in $R$, and if $Z$ is a closed set of capacity zero, then, for $P$ in $R$,

$$
\liminf _{Q \rightarrow P, Q} \Omega f(Q)=\Omega f(P) .
$$

3.9. Proofs of 3.2-3.8. We can write $g(P, Q)=h(P, Q)-\log P Q$, where $h(P, Q)$ is harmonic in $P$, for each $Q$, and is symmetric in $P, Q$. Then

$$
\Omega f^{+}(P)=-\frac{1}{2 \pi} \iint_{R} f^{+}(Q) h(P, Q) d Q+\frac{1}{2 \pi} \iint_{R} f^{+}(Q) \log P Q d Q .
$$

Let these two integrals be denoted by $\phi_{1}(P)$ and $\phi_{2}(P)$ respectively. The function $\phi_{1}$ is clearly continuous in $R$. Since

$$
\Delta_{r} h(P, Q)=\frac{1}{2 \pi r} \int_{C(P, r)} h(M, Q) d s_{M}-h(P, Q)=0,
$$

we have, by Fubini's theorem on the change of order of integration,

$$
\Delta_{r} \phi_{1}(P)=-\frac{1}{2 \pi} \iint_{R} f^{+}(Q) \Delta_{r} h(P, Q) d Q=0,
$$

which shows that $\phi_{1}$ is harmonic in $R$. Next, $\phi_{2}$ is subharmonic in $R$; this follows from $[5 ; 328]$, with $\mu(e)=\iint_{e} f^{+}(Q) d Q$. Thus 3.2 is proved.

By 3.2, $\Omega f^{+}$and $\Omega f^{-} \in L$ on $C(P, r)$ and on $J(P, r)[3 ; 4] .3 .3$ follows.

If $f$ is bounded above in $R, \Omega f^{+}(P)$ is finite at all $P$ of $R$. Hence $\Omega f(P)$ 
$>-\infty$ in $R$. Since $\Omega m$ is continuous in $R$ for any constant $m$, we may assume without loss of generality that $f(P) \leqq 0$ in $R$. Lower semi-continuity (and hence 3.4) follows from 3.2 and 2.1.

Next, (3.5.1) is a consequence of Fubini's theorem and the formula

$$
\Delta_{r} \log P Q=\frac{1}{2 \pi r} \int_{C(P, r)} \log M Q d s_{M}-\log P Q= \begin{cases}0 & (P Q>r), \\ \log r / P Q & (P Q \leqq r) .\end{cases}
$$

By 3.2, (3.5.2) holds for $f^{+}$and for $f^{-}[3 ; 8]$, and hence for $f$.

Noting that $\lim _{r \rightarrow 0}\left(1 / \pi r^{2}\right) \iint_{J(P, r)} f(Q) d Q=f(P)$ p. p. in $R[6 ; 118]$, we see that 3.2, (3.9.1), and (2.2.3) imply $\Lambda \Omega f^{+}(P)=f^{+}(P)$ p. p. in $R$. 3.6 follows.

To prove 3.7, we note that $f$ is bounded above in a neighborhood of $P$. Hence $\Omega f(P)$ is defined. Suppose first that $f(P)$ is finite. Let $\epsilon>0$ be arbitrary. There exists $\delta>0$ such that $f(Q) \leqq f(P)+\epsilon$ for $Q$ in $J(P, \delta)$. By (3.5.1) we have, for $0<r<\delta$,

$$
\Delta_{r} \Omega f(P) \leqq \frac{1}{2 \pi} \iint_{J(P, r)}(f(P)+\epsilon) \log \frac{r}{P Q} d Q=\frac{1}{4} r^{2}(f(P)+\epsilon) .
$$

Hence $\Lambda^{*} \Omega f(P) \leqq f(P)+\epsilon$, which implies $\Lambda^{*} \Omega f(P) \leqq f(P)$. Next, suppose $f(P)$ $=-\infty$. Let $m>0$ be arbitrary. There exists $\delta>0$ such that $f(Q) \leqq-m$ for $Q$ in $J(P, \delta)$. Proceeding as above, we see that $\Lambda^{*} \Omega f(P) \leqq-m$, which implies $\Lambda^{*} \Omega f(P)=-\infty$.

To prove 3.8, we note that $\Omega f$ is defined in $R$, and that

$$
\liminf _{Q \rightarrow P, Q \notin z} \Omega f(Q) \geqq \liminf _{Q \rightarrow P} \Omega f(Q) \geqq \Omega f(P)
$$

(by 3.4). Thus, if 3.8 is false, there exists a number $m$ such that

$$
\liminf _{Q \rightarrow P, Q \in Z} \Omega f(Q)>m>\Omega f(P) \text {. }
$$

For $Q$ sufficiently near to $P, Q \notin Z$, we have then $\Omega f(Q) \geqq m$. By (2.3.1), $Z$ is of measure zero on every circle; hence

$$
\liminf _{r \rightarrow 0} \frac{1}{2 \pi r} \int_{C(P, r)} \Omega f(Q) d s_{Q} \geqq m>\Omega f(P),
$$

contrary to $(3.5 .2)$.

\section{Proof of MaIN RESUlts}

In the proof of Theorem I we shall make use of the following theorem.

4.1. Vitali-Carathéodory Theorem. Let $f$ be defined in $R, f \in L$ on $R$. Then there exist functions $u_{1}, u_{2}, \cdots, v_{1}, v_{2}, \cdots$, defined in $R$, with the following properties:

(a) $u_{1}(P) \leqq u_{2}(P) \leqq \cdots \leqq f(P) \leqq \cdots . v_{2}(P) \leqq v_{1}(P)$ in $R$; 
(b) each $u_{n}$ is bounded above and is upper semi-continuous in $R$, each $v_{n}$ is bounded below and is lower semi-continuous in $R$;

(c) $u_{n} \in L, v_{n} \in L$ on $R, n=1,2,3, \cdots$;

(d) $u_{n}(P) \rightarrow f(P), v_{n}(P) \rightarrow f(P)$ as $n \rightarrow \infty$, at almost all points $P$ of $R$.

For the proof we refer to $[6 ; 75]$.

4.2. Proof of Theorem I. Let $u$ be one of the upper semi-continuous functions associated with $y$ in $R$, in the sense of the Vitali-Caratheodory Theorem. Put

$$
W(P)=F(P)-\Omega u(P)
$$

$(P$ in $R)$.

By 3.4, $W$ is upper semi-continuous and $<+\infty$ in $R$. Applying 3.7 to $u$, we have, for $P$ in $R-R \cdot Z$,

$$
\begin{aligned}
\Lambda^{*} W(P) & \geqq \Lambda^{*} F(P)-\Lambda^{*} \Omega u(P) \geqq \Lambda^{*} F(P)-u(P) \\
& \geqq \Lambda^{*} F(P)-y(P) \geqq 0 .
\end{aligned}
$$

Here we used assumption (ii) and the first half of assumption (i) of Theorem I.

By 3.2, $W(P)$ is finite on a dense set in the domain $R-R \cdot Z$ (see (2.3.2)). Thus, by (2.1.2), $W$ is subharmonic in $R-R \cdot Z$.

Let $M \in R \cdot Z$. By (2.3.2), there exists a simple closed curve $\Gamma$ in $R$ whose interior $G$ contains $M$, such that $\Gamma \cdot Z=0$. Being upper semi-continuous and less than $+\infty$ in $R, W$ is bounded above in $G$. Moreover, by 3.8,

$$
\begin{aligned}
\limsup _{Q \rightarrow M, Q \operatorname{fup}_{z}} W(Q) & =\lim _{Q \rightarrow M} F(Q)-\liminf _{Q \rightarrow M, Q \bigoplus_{Z}} \Omega u(Q) \\
& =F(M)-\Omega u(M)=W(M) .
\end{aligned}
$$

Since $G \cdot Z$ is closed, we can now apply (2.3.3), and conclude that $W$ is subharmonic in $G$, and thus in $R$. By $2.2, \Lambda W(P)$ is finite p. p. in $R$. By 3.6,

$$
\Lambda F(P)=\Lambda W(P)+\Lambda \Omega u(P)=\Lambda W(P)+u(P)
$$

Since $R$ is an arbitrary bounded domain in $D$, it follows that $\Lambda F(P)$ is finite p. p. in $D$. Similarly, 2.2 shows that $\Lambda F \in L$ on any closed subset of $R$, and hence on any closed subset of $D$. By 3.3, (1.2.1) holds p. p. in $D$. We have thus proved (a), (b), and (c) of Theorem I.

Next, put

$$
f(P)=\left(\Lambda^{*} F(P)+\Lambda_{*} F(P)\right) / 2
$$

at all points $P$ of $D$ at which the sum in (4.2.5) is defined, and put $f(P)=0$ at all other points of $D$. Then

$$
\begin{aligned}
\Lambda_{*} F(P) & \leqq f(P) \leqq \Lambda^{*} F(P) \\
f(P) & =\Lambda F(P)
\end{aligned}
$$

$(P$ in $D)$,

Let $u_{1}, u_{2}, \cdots, v_{1}, v_{2}, \cdots$, be functions associated with $f$ and $R$ in the 
sense of 4.1. Let $E$ be the set on which both $\Omega u_{1}(P)$ and $\Omega v_{1}(P)$ are finite. It follows, by (a) of 4.1 , that $\Omega u_{n}(P), \Omega v_{n}(P)(n=1,2,3, \cdots)$, as well as $\Omega f(P)$, are finite for $P$ on $E$. By $3.3, E$ contains almost all points of $R$. Let $J(M, r) \subset R$, with $M$ in $E$. This choice of $M$ assures that all integrals used in the remainder of this proof are finite. Put

$$
W_{n}(P)=F(P)-\Omega u_{n}(P)
$$

$$
(P \in R ; n=1,2,3, \cdots) .
$$

Proceeding as in (4.2.2) and (4.2.3), with $u_{n}$ and $f$ in place of $u$ and $y$, we see that $W_{n}$ is subharmonic in $R$. By (2.1.1)
$\Delta_{r} W_{n}(M) \geqq 0$
$(n=1,2,3, \cdots)$

By (3.5.1), (4.2.8), and (4.2.9),

$$
\Delta_{r} F(M) \geqq \frac{1}{2 \pi} \iint_{J(M, r)} u_{n}(Q) \log \frac{r}{P Q} d Q \quad(n=1,2,3, \cdots) .
$$

The sequence $\left\{u_{n}(P)\right\}$ converges to $f(P)$ monotonically p. p. in $R$. Hence we may pass to the limit in (4.2.10), and obtain

$$
\Delta_{r} F(M) \geqq \frac{1}{2 \pi} \iint_{J(M, r)} f(Q) \log \frac{r}{P Q} d Q .
$$

In the same manner, working with the functions $v_{n}(P)$, we obtain (4.2.11) with the inequality reversed. Hence the equality sign holds in (4.2.11), and comparison with (3.5.1) shows that

$$
\Delta_{r}(F(M)-\Omega f(M))=0 .
$$

By 3.3, the function $F-\Omega f$ thus satisfies all hypotheses of 2.4. Hence there exists a function $H$, harmonic in $R$, such that

$$
F(P)=\Omega f(P)+H(P)
$$

(p. p. in $R$ ).

Now let $N$ be a point in $R$ at which (1.2.1) holds. By (4.2.7), $\Omega f(N)$ is finite. Since $F$ and $H$ are continuous in $R$, we have

$$
\begin{aligned}
F(N)-H(N) & =\lim _{r \rightarrow 0} \frac{1}{\pi r^{2}} \iint_{J(N, r)}(F(Q)-H(Q)) d Q \\
& =\lim _{r \rightarrow 0} \frac{1}{\pi r^{2}} \iint_{J(N, r)} \Omega f(Q) d Q=\Omega f(N),
\end{aligned}
$$

the last equality being a consequence of (3.5.2).

4.3. We have now proved Theorem $I$, except for the assertion concerning the boundary values of $H$.

Put $u_{1}(P)=\Omega f^{+}(P), u_{2}(P)=\Omega f^{-}(P)$, for $P$ in $R$. By 3.2, $u_{1}$ and $u_{2}$ are subharmonic in $R$; the least harmonic majorants of $u_{1}$ and $u_{2}$ in $R$ are both zero $[5 ; 357]$. 
Fix a point $M$ in $R$. Choose a sequence $\left\{R_{n}\right\}$ of domains with polygonal boundaries $B_{n}$, such that $M \in R_{1}, R_{1} \subset R_{2} \subset \cdots \subset R, R_{n} \rightarrow R$ as $n \rightarrow \infty$. The functions $u_{1}$ and $u_{2} \in L$ on $B_{n}$, since they are subharmonic [2;14]. Since $\Omega f=u_{1}-u_{2}$, this shows that $\Omega f$ exists p. p. on $B_{n}$. We have shown that (4.2.13) holds wherever $\Omega f$ is finite. Hence (4.2.13) holds at almost all points of $B_{n}$, which implies

$$
H_{n}(M)=h_{n}^{(1)}(M)-h_{n}^{(2)}(M)+H(M),
$$

where $H_{n}$ is harmonic in $R_{n}$ and coincides with $F$ on $B_{n}$, and $h_{n}^{(1)}, h_{n}^{(2)}$, are the best harmonic majorants of $u_{1}, u_{2}$, in $R_{n}$.

(For the concepts of best harmonic majorants (B.H.M.) and least harmonic majorants (L.H.M.) of a subharmonic function in a given domain we refer to $[4 ; 334],[5 ; 357-358]$. Radó $[3 ; 39]$ proved that the L.H.M. is equal to the B.H.M. for Dirichlet domains. Since the B.H.M. is defined for Dirichlet domains only, it would seem that Radó's theorem has made the concept of B.H.M. superfluous.)

Since $\lim h_{n}^{(1)}(M)=0=\lim h_{n}^{(2)}(M)$ as $n \rightarrow \infty$ [5;358], (4.3.1) implies that $\lim H_{n}(M)=H(M)$. This completes the proof of Theorem I.

4.4. Proof of Theorem II. All hypotheses of Theorem I are evidently satisfied (we can take $y=0$ ). To show that (1.3.1) holds everywhere, it is sufficient, by (d) of Theorem I, to show that $\Omega f(P)>-\infty$ for $P$ in $R$, where $f=\Lambda^{*} u \geqq 0$. By $3.4, \Omega f$ is upper semi-continuous in $R$, hence the equation $\Omega f(P)$ $=-\infty$ would imply $\lim \Omega f(Q)=-\infty$ as $Q \rightarrow P$, and $\Omega f$ would not be equal to the continuous function $u-H$ almost everywhere in $R$.

The remaining assertions of Theorem II were proved by Riesz $[5 ; 357]$ for the Stieltjes integral representation. Hence they evidently hold in our case as well.

\section{BIBLIOGRAPHY}

1. M. Brelot, Sur la structure des ensembles de capacité nulle, C. R. Acad. Sci. Paris vol. 192 (1931) pp. 206-208.

2. - Étude des fonctions sousharmoniques au voisinage d'un point, Paris, 1934.

3. T. Radó, Subharmonic functions, Ergebnisse der Mathematik, vol. 5, no. 1, Berlin, 1937.

4. F. Riesz, Sur les fonctions subharmoniques et leur rapport à la thêorie du potentiel, Acta Math. vol. 48 (1926) pp. 329-343.

5. - - Sur les fonctions subharmoniques et leur rapport à la théorie du potentiel, Acta Math. vol. 54 (1930) pp. 321-360.

6. S. Saks, Theory of the integral, 2d. ed., Warsaw, 1937.

7. - On the operators of Blaschke and Privaloff for subharmonic functions, Rec. Math. (Mat. Sbornik) vol. 51 (1941) pp. 451-456.

8. N. Wiener, Certain notions in potential theory, Journal of Mathematics and Physics vol. 3 (1924) pp. 24-51.

9. A. Zygmund, Trigonometrical series, Warsaw, 1935.

DURe University, DURHAM, N. C. 